\begin{tabular}{|c|c|}
\hline Title & Improvement of transcutaneous fluorescent images with a depth-dependent point-spread function \\
\hline Author(s) & Shimizu, Koichi; Koji, Tochio; Kato, Y uji \\
\hline Citation & $\begin{array}{l}\text { A pplied Optics, 44(11), 2154.2161 } \\
\text { https://doi.org/10.1364/A 0.44.002154 }\end{array}$ \\
\hline Issue Date & $2005-04$ \\
\hline Doc URL & http://hdl.handle.net/2115/643 \\
\hline Rights & (c) 2005 Optical Society of A merica \\
\hline Type & article \\
\hline File Information & 4411_p2154-2161.pdf \\
\hline
\end{tabular}

Instructions for use 


\title{
Improvement of transcutaneous fluorescent images with a depth-dependent point-spread function
}

\author{
Koichi Shimizu, Koji Tochio, and Yuji Kato
}

\begin{abstract}
The point-spread function (PSF) for transcutaneous fluorescent imaging was obtained as an analytical solution in a closed form. It is applicable to cases in which the optical property of the image-blurring turbid medium is considered to be fairly homogeneous. We proposed a technique to improve a transcutaneous image by using depth-dependent PSF. Contrast of the fluorescent image was improved for depths of $1-15 \mathrm{~mm}$ in a scattering medium $\left(\mu_{s}{ }^{\prime}=1 / \mathrm{mm}\right)$. The visible depth was more than doubled with this technique. An experiment with a rat demonstrated considerable improvement of a transcutaneous image of the cerebral vein at a specified depth. The spread image of the heart was reduced to the correct size by use of the PSF with the actual depth of the heart. (C) 2005 Optical Society of America

OCIS codes: $170.3880,170.3660,170.7050,170.3010,100.3020,100.1830$.
\end{abstract}

\section{Introduction}

The feasibility of transillumination imaging of the human body has been acknowledged since the 19th century. ${ }^{1-3}$ In the 1980 s and 1990 s numerous studies reported applications of transillumination imaging with lasers and solid-state detectors. However, those methods have not been widely used in common practice. Poor spatial resolution caused by the strong scattering by body tissues is a major reason for its lack of use.

In contrast to the active development of diffuse optical tomography and optical coherent tomography, recent studies that specifically address transillumination imaging have been few. Nevertheless, the importance of transillumination imaging has been indicated in new applications: One is noninvasive measurement of physiological functions in experimental animals. ${ }^{4,5}$ The use of large animals in experiments has become difficult. Moreover, noninvasive techniques have been required for animal welfare. ${ }^{6}$ Another application is the use of the transillumination technique for transcutaneous fluorescent

K. Shimizu (shimizu@bme.ist.hokudai.ac.jp) and Y. Kato are with the Graduate School of Information Science and Technology, Hokkaido University, Sapporo 060-0814, Japan. K. Tochio is with the Hakuju Institute for Life Science Company, Ltd., 1-37-5 Tomigaya, Shibuya-ku, Tokyo 151-0063, Japan.

Received 21 July 2004; revised manuscript received 12 December 2004; accepted 11 January 2005.

0003-6935/05/112154-08\$15.00/0

(C) 2005 Optical Society of America imaging. ${ }^{6-8}$ The use of fluorescent markers allows functional imaging at the genetic, cellular, and organ levels.

In these areas the images of the absorbing part or the fluorescent part inside a mouse or a rat are often recorded through surface tissue. It is common to observe the existence or the approximate position of such parts. Generally, optimization with respect to scattering suppression is not considered. For that reason, the obtained image is typically blurred because of the scattering by body tissues. Surgery is required to remove the surface tissue and thereby reveal the fine structure and the depth of the absorbing or fluorescent part.

We have pointed out the importance of functional transillumination imaging and have developed a technique to suppress that scattering effect.4,9-11 Using this technique, we have demonstrated the feasibility of the functional transillumination imaging of rat brain ${ }^{9,10}$ for the first time. This technique is applicable only to body portions for which we can obtain transmitted light that is sufficiently strong for imaging.

For thick parts, we must use reflection measurement geometry, or a fluorescent method..$^{12}$ Even with this geometry, the fluorescent source image is blurred by scattering resulting from the interposition of tissue between the light source and the skin surface. The blurring effect in imaging through body tissue has been studied extensively; many reports have addressed spatial resolution in transillumination imaging. ${ }^{13-15}$ Spatial resolution can be evaluated in terms of the spatial point-spread function (PSF). We 


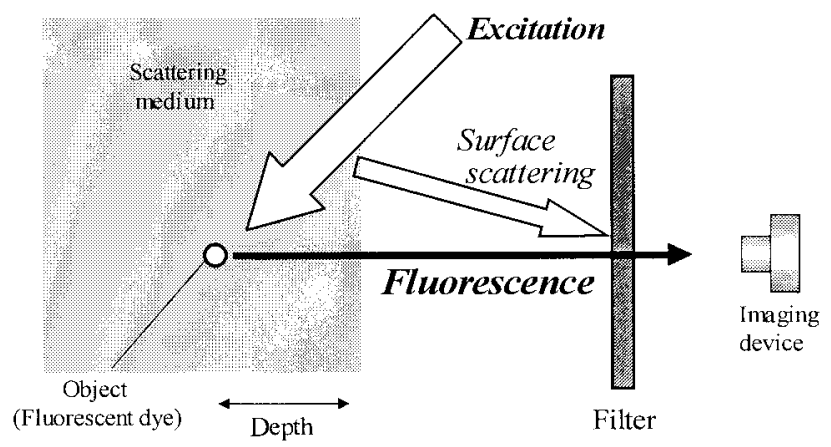

Fig. 1. Principle of transcutaneous fluorescent imaging.

can suppress the scattering effect by deconvolution with the PSF. The PSF for imaging through a scattering medium has been obtained by use of the random-walk theory. ${ }^{16,17}$ The significance of the PSF in a simple analytical form has been shown in experiments. ${ }^{18}$ However, this PSF was for imaging in a transmission measurement geometry. Therefore it is not applicable to transcutaneous fluorescent imaging, in which the light source is in the scattering medium.

This paper describes our attempt to derive the PSF for fluorescent imaging from a different theory of light propagation in random media. We try to obtain the solution in a closed analytical form for ease of use in inverse problems. Using the solution, we demonstrate the usefulness of depth-dependent PSF for transcutaneous fluorescent imaging ${ }^{19}$ with a view toward application to noninvasive functional imaging of a human body and an experimental animal.

\section{Analysis for the Transcutaneous Point-Spread Function}

\section{A. Transcutaneous Imaging of Fluorescence}

In conventional transillumination imaging, light is illuminated from behind the object. The image is obtained with the imaging device, e.g., a CCD, from the obverse side of the object. The usefulness of such transillumination imaging has been recognized and used in various applications. However, some limitations exist for this technique. It is applicable only to body portions for which transmitted light is available. Even if a light-absorbing structure exists close to the surface, we cannot obtain a transilluminated image when the body is too thick to transmit sufficient light for imaging.

Reflection measurement geometry can be used for such cases: The light source and the imaging device are placed on the same side of the object. By carefully adjusting the illumination condition, we can obtain the absorption distribution near the body surface. However, it is generally difficult to apply this technique to a mammalian body because of strong scattering at the skin surface. The use of a fluorescent material as a contrast agent is one solution for this problem. Figure 1 shows the principle of this technique - transcutaneous fluorescent imaging. We

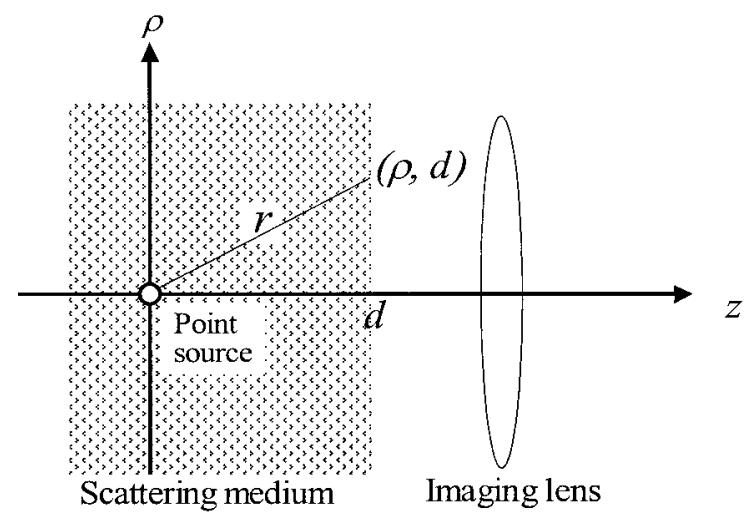

Fig. 2. Geometry of the theoretical model.

illuminate the excitation light to an object body. The fluorescent light is excited in the body and is transmitted through the surface tissue. The wavelength of the fluorescent light is generally different from that of the excitation light. Therefore, the scattered light at the surface can be eliminated with an optical filter. In this way, we can attain transcutaneous fluorescent imaging using reflection measurement geometry. ${ }^{12,19}$

\section{B. Derivation of the Point-Spread Function}

Images obtained through transcutaneous imaging are blurred because of the light scattering by tissue between the fluorescent source and the skin surface. Even with this blurred image we can still know the existence of the fluorescent source and its crude localization. However, we often require better spatial resolution to distinguish organs or to understand the shape of the fluorescent distribution. We can suppress the scattering effect by deconvolving the blurred image with the PSF if we know the degree of blurring as a PSF. The PSF can be obtained in the measurement, but it is not easy to measure all PSFs for every possible condition. Therefore we must first derive the PSF theoretically.

Figure 2 illustrates the geometry of the theoretical model. A point light source is placed at the origin of the circularly symmetrical cylindrical coordinate system $(\rho, z)$. The scattering medium fills the half space $(z \leq d)$. The PSF is defined as the spatial distribution of light intensity at the surface of the scattering me$\operatorname{dium}(z=d)$. The point source radiating total power $P_{0}$ uniformly in all directions is expressed as

$$
\varepsilon(\bar{r})=\frac{P_{0}}{4 \pi} \delta(\bar{r}),
$$

where $\bar{r}$ is the position vector. We can divide the light intensity at the surface into two parts. They are the direct light component attenuated by the scattering and the absorption of the scattering medium, and the diffuse light component. The flux of the reduced direct light is given as

$$
\bar{F}_{r i}(\bar{r})=P_{0} \frac{\exp \left[-\left(\mu_{s}+\mu_{a}\right) r\right]}{4 \pi r^{2}} \hat{r},
$$


where $\mu_{s}, \mu_{a}$, and $\hat{r}$ are the scattering coefficient, the absorption coefficient, and the unit vector in the direction of $\bar{r}$, respectively. The scattering coefficient of surface tissue is typically $15-30 \mathrm{~mm}^{-1}$ in the nearinfrared wavelength. ${ }^{20}$ Therefore this component reduces rapidly in the practical range of the distance from the source to the surface $(r>0.2 \mathrm{~mm})$.

The diffuse component is obtained by application of the diffusion approximation to the equation of transfer. ${ }^{21}$ The diffusion equation for the point source is

$$
\nabla^{2} U_{d}(\bar{r})-\kappa_{d}^{2} U_{d}(\bar{r})=-(3 / 4 \pi) P_{0}\left(\mu_{s}{ }^{\prime}+\mu_{a}\right) \delta(\bar{r}),
$$

where $\kappa_{d}^{2}=3 \mu_{a}\left(\mu_{s}{ }^{\prime}+\mu_{a}\right)$ and $\mu_{s}{ }^{\prime}=(1-g) \mu_{s} . U_{d}(\bar{r})$, $\mu_{s}{ }^{\prime}$, and $g$ are the average diffuse intensity, the reduced scattering coefficient, and the scattering anisotropy parameter, respectively. Solving this equation, $U_{d}(\bar{r})$ and the diffuse flux $\bar{F}_{d}(\bar{r})$ are given as

$$
\begin{aligned}
& U_{d}(\bar{r})=\frac{3 P_{0}}{(4 \pi)^{2}}\left(\mu_{s}{ }^{\prime}+\mu_{a}\right) \frac{\exp \left(-\kappa_{d} r\right)}{r}, \\
& \bar{F}_{d}(\bar{r})=\frac{P_{0}}{4 \pi}\left(\kappa_{d}+\frac{1}{r}\right) \frac{\exp \left(-\kappa_{d} r\right)}{r} \hat{r} .
\end{aligned}
$$

Using these results, we finally obtain the PSF as

$$
\begin{aligned}
P(\rho)= & \frac{3 P_{0}}{(4 \pi)^{2}}\left\{\left(\mu_{s}{ }^{\prime}+\mu_{a}\right)+\left[\kappa_{d}+\frac{1}{\left(\rho^{2}+d^{2}\right)^{1 / 2}}\right]\right. \\
& \left.\times \frac{d}{\left(\rho^{2}+d^{2}\right)^{1 / 2}}\right\} \frac{\exp \left[-\kappa_{d}\left(\rho^{2}+d^{2}\right)^{1 / 2}\right]}{\left(\rho^{2}+d^{2}\right)^{1 / 2}},
\end{aligned}
$$

where $\rho$ and $d$ are, respectively, the radial distance from the $z$ axis and the depth of the point source from the surface of the scattering medium.

Diffusion approximation is valid when the thickness of the scattering medium is much greater than the average mean-free path length or $1 / \mu_{s}{ }^{\prime}$. Thus we must be careful to apply Eq. (6) when $\left(\rho^{2}+d^{2}\right)^{1 / 2}$ is not much larger than $1 / \mu_{s}{ }^{\prime}$. This is examined in the experiment in the latter part of Section 3. In this derivation the scattering medium is assumed as homogeneous. In many practical applications the scattering coefficient of intervening tissue is almost homogeneous. We do not usually attempt to render an image for the fluorescence behind a large lightabsorbing object.

\section{Measurement of the Point-Spread Function}

Through the above theoretical analysis, we obtained the PSF in a closed analytical form. This means that we can obtain the PSF if we know or can estimate the geometrical and scattering parameters of the scattering medium. In particular, the depth dependence of the PSF in a scattering medium plays an important role in practical applications. For imaging through skin tissue, we often do not know the exact depth of the fluorescent source. We can process the transcu-

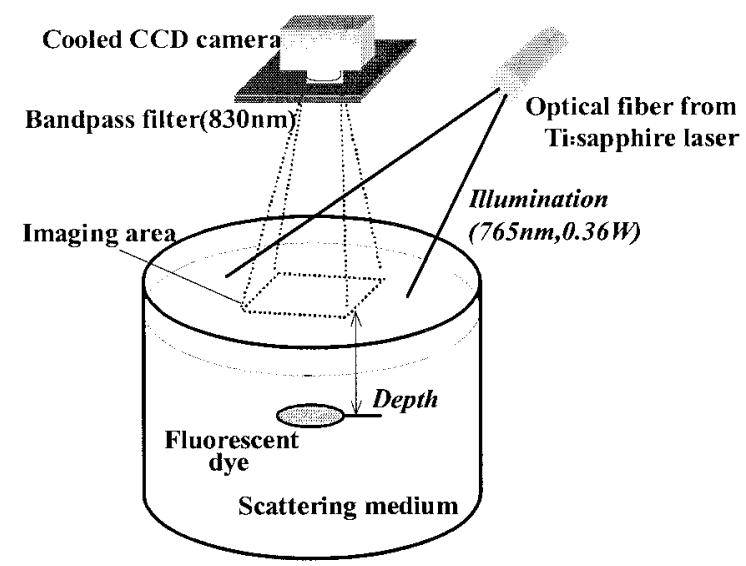

Fig. 3. Experimental setup for PSF measurement.

taneous image using PSF with a variable depth parameter if we have the PSF as a function of the depth. By scanning the parameter, we can determine the depth of the fluorescent source by using the focusfinding algorithm if the source is distributed at a certain depth. When the source is distributed over some depth range, we may be able to visualize the three-dimensional structure by using the confocal mapping algorithm in confocal laser microscopy. We must have the correct values for the parameters of Eq. (6) if we require the absolute depth by using this technique. This is the limitation of the proposed technique. However, as in most cases, when the relative depth or approximate depth suffices, we can obtain it effectively by using this technique.

The PSF was measured in a model experiment to examine the appropriateness of the above solution and the validity of this principle. Figure 3 illustrates the experimental setup schematically. A point fluorescent source is ideal to measure the PSF, but it is difficult to realize in a practical measurement. Therefore we obtained the PSF by means of a deconvolution operation. A thin disk container $(13-\mathrm{mm}$ diameter, $0.8-\mathrm{mm}$ height) filled with a fluorescent medium was placed in a scattering medium with the circular face oriented upward to the CCD camera. A mixture of Indocyanine Green (ICG) and dimethyl sulfoxide (DMSO) solution was used as the fluorescent medium. ${ }^{22}$ The scattering medium was an aqueous solution of Intralipid $\left(\mu_{s}{ }^{\prime}=1.0 / \mathrm{mm}\right)$. The depth $d$ was measured from the surface of the scattering medium and varied from 1 to $15 \mathrm{~mm}$. Excitation light from a Ti:sapphire laser (765-nm wavelength) was guided through an optical fiber (multimode, $100-\mu \mathrm{m}$ core diameter). The laser light illuminated the fluorescent object in the scattering medium. The fluorescent light image was captured by means of a cooled CCD camera through an optical bandpass filter $(830-\mathrm{nm}$ central wavelength, 10-nm FWHM bandwidth).

As the first step, a fluorescent image with a known shape (a circular face of the fluorescent disk) was recorded through clear water. Then the water was replaced with a scattering medium; the fluorescent image was then recorded with the same imaging sys- 


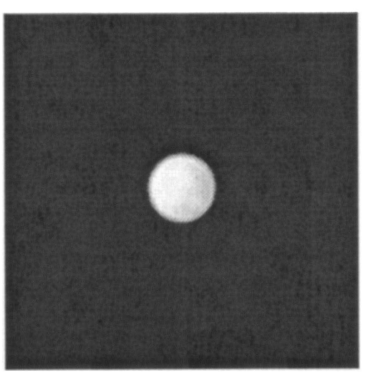

(a)

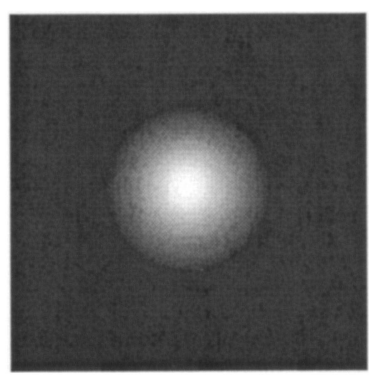

(b)
Fig. 4. Measured image of fluorescent source at 5-mm depth: (a) in clear water, (b) in scattering medium.

tem. Imaging through the scattering medium was repeated at different depths of the fluorescent source. The PSF at specific depth was obtained through twodimensional deconvolution of the blurred image in a scattering medium with the image in clear water. In the deconvolution we obtained the two-dimensional PSF. Considering the cylindrical symmetry of the measurement system, the final PSF was obtained as a function of the radial distance by averaging the two-dimensional PSF over the azimuthal direction.

Figures 4 and 5 show measured images and typical examples of the obtained PSF. Figure 6 shows the spread (FWHM) of the measured PSF as a function of the depth of the fluorescent source. The solid curve shows the calculated PSF by use of the derived Eq. (6). Both the relative shape of the curve and absolute

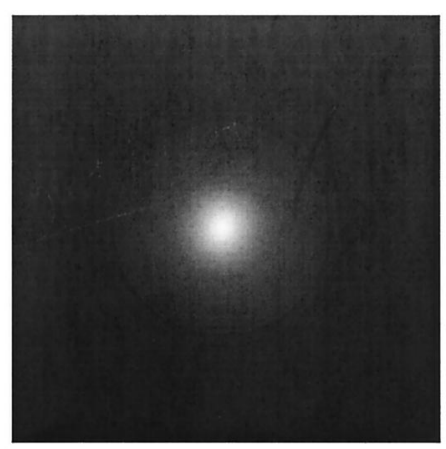

(a)

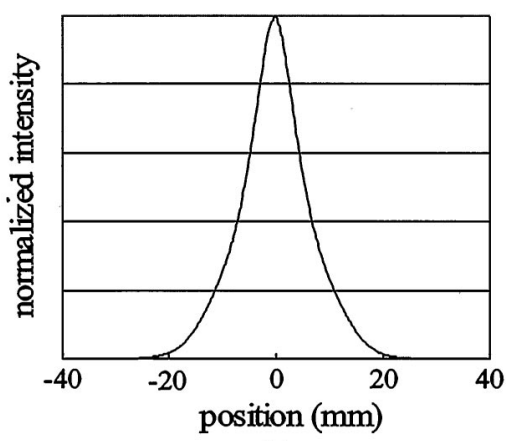

(b)

Fig. 5. PSF obtained in the deconvolution of measured data: (a) two-dimensional PSF for 5-mm depth, (b) averaged PSF over all directions.

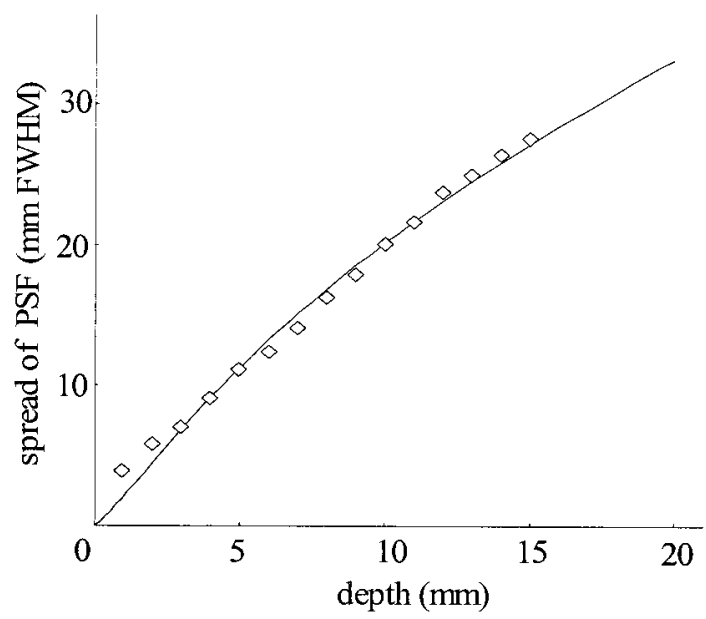

Fig. 6. Depth dependence of measured PSF spread. Diamonds and curve are measurement and theoretical calculation, respectively.

value of the spread agreed well with the measured values. As previously mentioned, the validity of the derived equation with a small depth was in question. This result suggests its validity from a 1-mm depth.

\section{Improvement by Use of the Depth-Dependent Point-Spread Function}

Through theoretical analysis we derived the PSF as a function of the object depth. Experimental analysis confirmed the validity of the depth-dependent PSF for the scattering medium that was similar to the body tissue. Using this depth-dependent PSF, we can expect improvement in fluorescent imaging through a scattering medium. The degree of improvement was evaluated in fluorescent imaging by use of a model phantom to examine the effectiveness of the proposed technique. The experimental setup was similar to that shown in Fig. 3 except for the fluorescent object. In this case two circular disk containers $(13-\mathrm{mm}$ diameter, $0.8-\mathrm{mm}$ height each, $23 \mathrm{~mm}$ apart center to center) filled with ICG and DMSO solution were placed in the scattering medium with the circular faces oriented toward the CCD camera.

Figure 7 shows typical examples of the images before and after the deconvolution operation. They are cases with depths of 5 and $9 \mathrm{~mm}$. The effect of scattering was greatly reduced with the appropriate PSF at each depth. Figure 8 shows the intensity profile along the line between the centers of the two circular faces of the disks. The deconvolution method is known to amplify the noise in an original figure. However, as this figure shows, the signal-to-noise ratio did not increase markedly with the spread of this PSF.

The degree of improvement was evaluated by the contrast of the image. Figure 9 shows the result. The contrast dropped rapidly as the depth of the fluorescent object increased in the scattering medium. Contrast was improved by depth-dependent PSF in all depths measured in the experiment. The average contrast between 1 - and $15-\mathrm{mm}$ depths was more than 

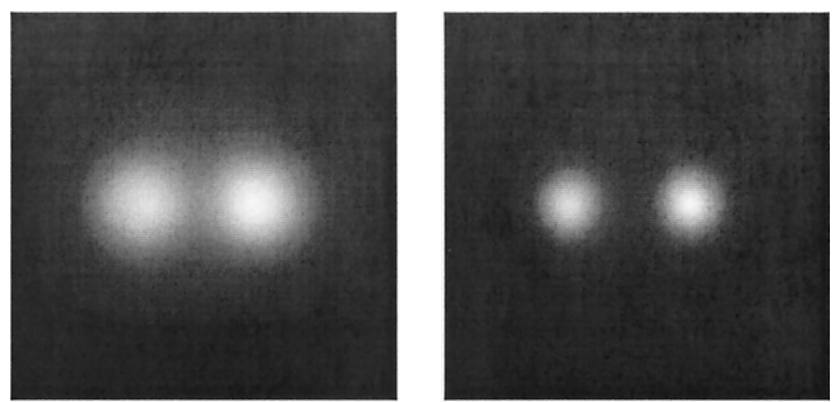

(a)
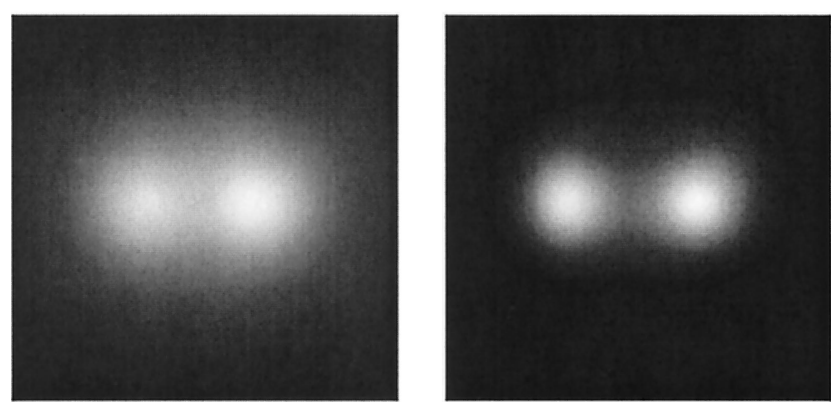

(b)

Fig. 7. Improvement by depth-dependent PSF (left and right figures are those before and after deconvolution, respectively): (a) 5-mm depth, (b) 9-mm depth.

twice the original value, indicating that the visible depth in the scattering medium is more than doubled by applying this technique. This experimental study confirms the effectiveness of the proposed technique.

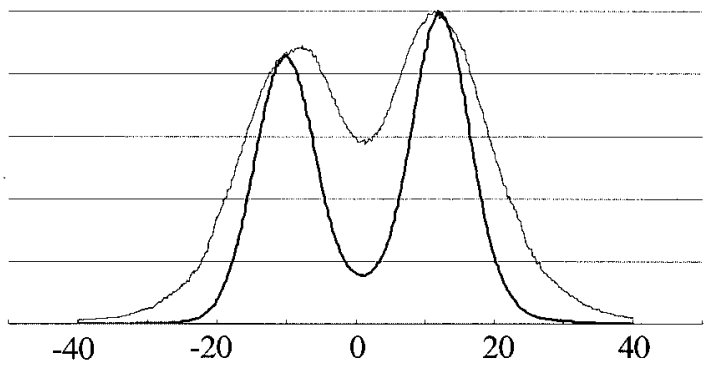

(a)

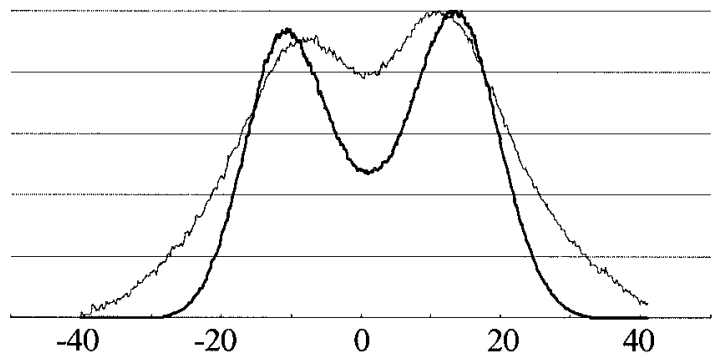

(b)

Fig. 8. Intensity profiles of fluorescent images before (light curve) and after (dark curve) improvement: (a) 5-mm depth, (b) 9-mm depth.

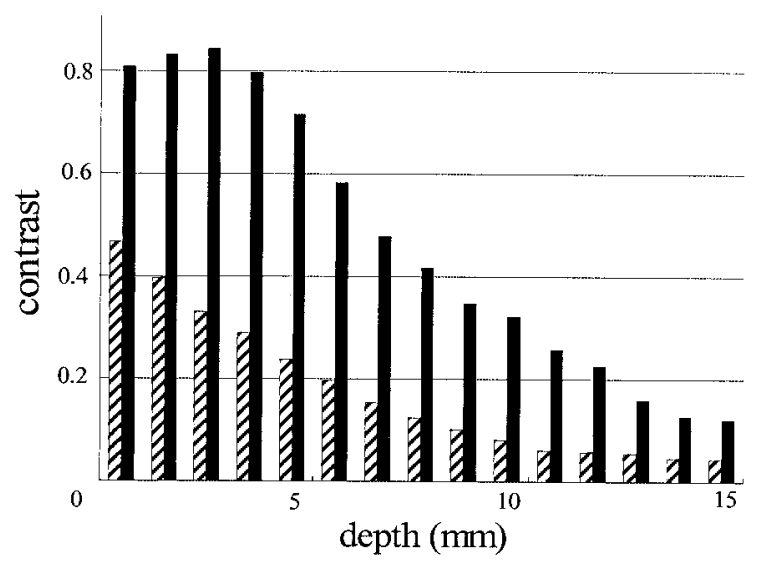

Fig. 9. Improvement in the contrast of the fluorescent image. Striped bars, the contrasts before improvement; solid bars, the contrasts after improvement.

\section{Improvement in Animal Imaging}

An animal experiment was conducted to examine the applicability of the proposed technique to practical imaging. Figure 10 illustrates the experiment schematically. The instruments used were the same as in Fig. 3. An experimental animal, a rat (Wistar, 4-week-old female, 50-g weight), was used. For imaging it was anesthetized by intra-abdominal administration of pentobarbital. Fur at the imaging surface was eliminated by use of hair-remover cream. The ICG was diluted with distilled water prepared for infusion and was injected into the rat's tail vein. Fluorescent images were recorded at the head area and the abdominal area.

Figure 11(a) shows the fluorescent image of the rat's head area. Major veins are visible on the brain surface. Finer structures, such as small veins, were hardly visible because of the scattering by body tissue. This image was processed with the depthdependent PSF. Figure 11(b) shows the processed image with the PSF of 0.53-mm depth. Sagittal and transverse veins appear more clearly than in Fig. 11(a). Figure 11(c) shows the intensity profiles along the horizontal line at the vertical position of $30 \mathrm{~mm}$. The increase in the contrast of the sagittal vein is

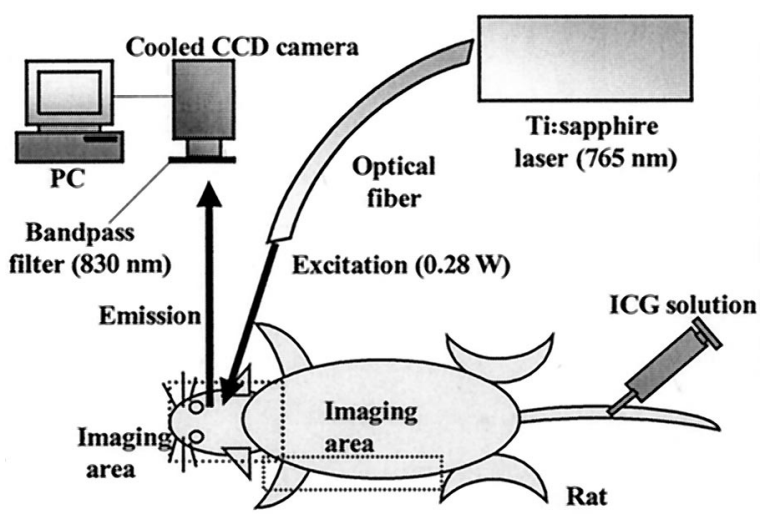

Fig. 10. Experimental setup for transcutaneous fluorescent imaging with a rat. 


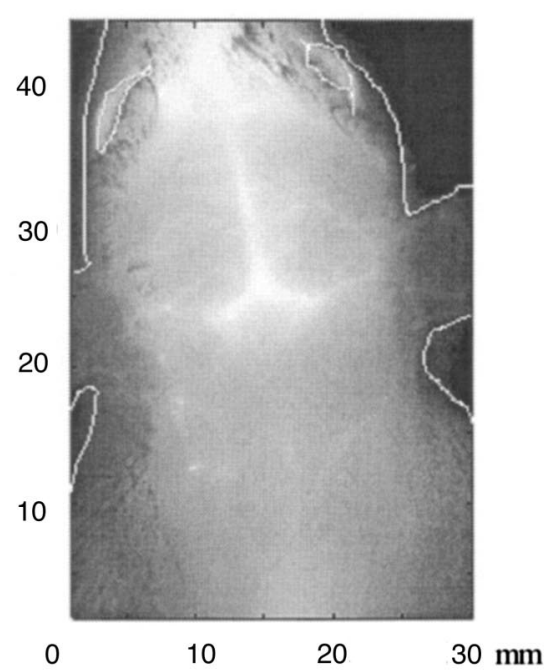

(a)

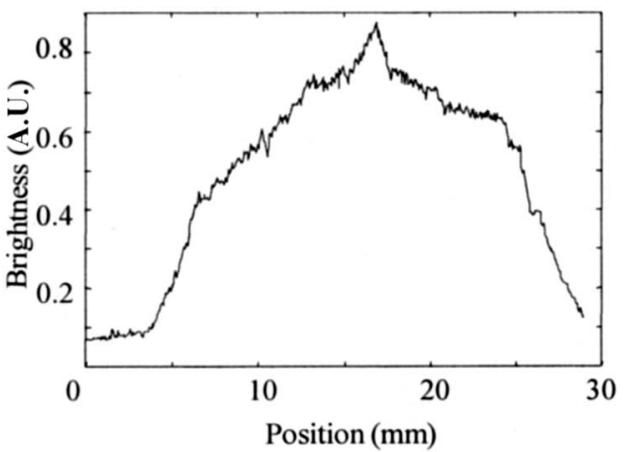

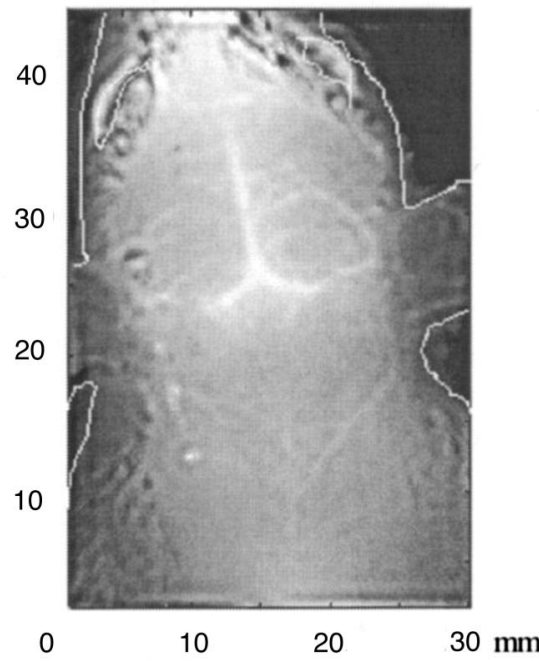

(b)

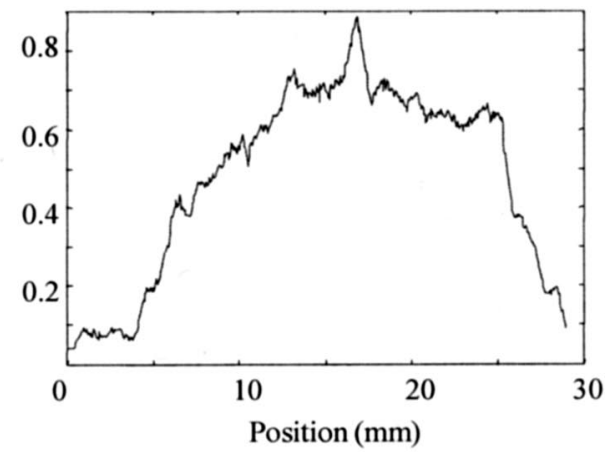

(c)

Fig. 11. Improvement in transcutaneous fluorescent image of a rat's head (a) before and (b) after deconvolution with a PSF of 0.53-mm depth. Unit of scale is millimeters. (c) Intensity profile along the horizontal line at $30 \mathrm{~mm}$.

apparent. The decrease of the signal-to-noise ratio resulting from deconvolution was not large.

Figure 12(a) shows the image at the abdominal area. We could detect a fluorescent source inside, but the distinction of organs was not easy. This image was processed with a depth-dependent PSF of $16-\mathrm{mm}$ depth; it is shown in Fig. 12(b). In this figure we were able to identify the heart from its size and position. Subsequent to these measurements, the animal was sacrificed according to the guidelines of euthanasia for laboratory animals. ${ }^{23}$ During anatomical dissection the depths of the cerebral vein and the heart from the surface were measured. General agreement between the best focal depth in the image processing and the anatomical depth was confirmed.

\section{Conclusions}

Imaging of a fluorescent source inside the body has been widely used in many fields of life science. Such images are markedly blurred because of strong scattering by body tissues when we observe the fluorescent light through the body tissue. In such cases removal of surface tissue is necessary to find the exact spatial distribution of the fluorescent source. We have developed a technique to suppress the effects of scattering on the analysis. The method is based on a deconvolution operation with PSF. The PSF depends strongly on optical properties of the scattering medium and imaging geometry such as the depth of the fluorescent source in the body. Thus we need to find the PSF as a function of such parameters.

We evaluated two kinds of light intensity from the point source to derive the PSF theoretically: direct light and diffused light. We found that the diffused light is predominant in our application. We applied diffusion approximation to the equation of transfer and derived the PSF as an analytical solution in a closed form. The simple analytical form of the PSF is useful to save computation time in solving inverse problems. The PSF was measured by means of the fluorescent source with a known geometry at various depths in the scattering medium to examine the correctness of the derived PSF. The spread of the measured PSF agreed well with that predicted in the derived theoretical solution.

Using the depth-dependent PSF, we can improve the fluorescent image in a scattering medium. The effectiveness of the proposed technique was exam- 


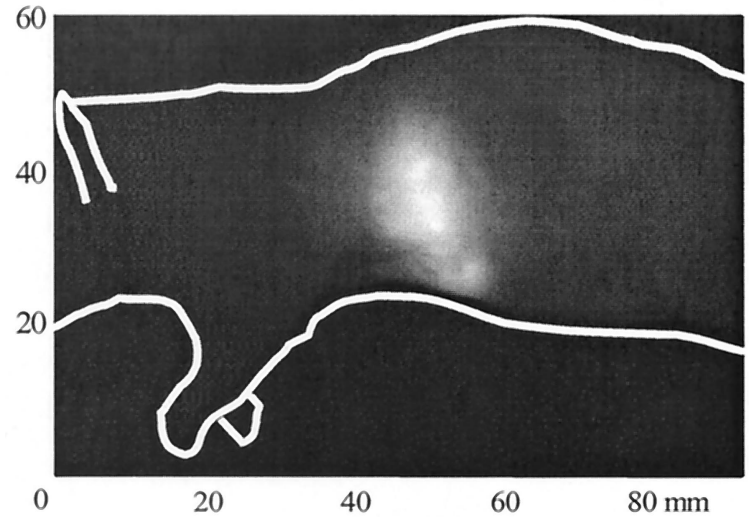

(a)

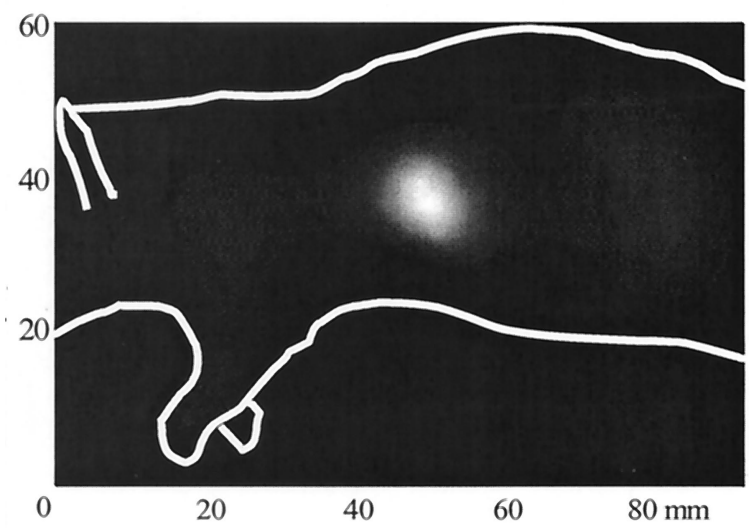

(b)

Fig. 12. Improvement in transcutaneous fluorescent image of a rat's abdomen (a) before and (b) after deconvolution with a PSF of 16-mm depth.

ined in a model experiment. Fluorescent images of known objects were obtained in a scattering medium. The contrast of the fluorescent image was improved for all depths of 1-15 $\mathrm{mm}$. With this technique, the visible depth was more than doubled.

Animal experiments were conducted to examine the applicability of the proposed technique to a living body. Fluorescent images of ICG were recorded transcutaneously in the head and the abdominal areas of a rat. Using the PSF with appropriate depth, the image of the specific vein at the depth was improved considerably. The spread image of the heart reduces to the correct size with the PSF at the depth of the heart. Through these analyses with the depthdependent PSF, the effectiveness of this technique was confirmed.

Using the proposed technique, we can improve the transcutaneous image of the fluorescent source distribution, provided we know the depth of the source. We can apply PSF with a variable depth to the image when the depth is indeterminate or when the fluorescent source is distributed over different depths. By measuring the contrast or the clarity of the target fluorescent source, we can focus upon the target. The $\mathrm{PSF}$ is given in the closed form of the analytical solution. Therefore it is not difficult to employ autofo- cusing principles in photography. In this way we can visualize the distribution of a fluorescent source with higher spatial resolution or with deeper visible range. If we know the homogeneous scattering coefficient and the object size $a$ priori, such as diameters of a specific vein or a specific organ, we can estimate the approximate depth of the object by applying the PSF with variable depth.

As described above, this technique is useful for better observation of the fluorescent light through the skin. It will also improve noninvasive diagnostics by use of fluorescent contrast agents in medicine. In animal experiments it can reduce the necessity of open surgery because it obviates the need to remove surface tissue for clear observation. Consequently, it will contribute to a reduction in the number of sacrificed animals in such experiments and the capability for long-term tracing of fluorescent sources, such as during growth periods of the animal.

This research was supported by a Grant-in-Aid for Scientific Research from the Japan Society for the Promotion of Science.

\section{References}

1. R. Bright, "Case CCV," in Reports of Medical Cases Selected with a View of Illustrating the Symptoms and Cure of Diseases by a Reference to Morbid Anatomy, Diseases of the Brain and Nervous System, (Longmans, Green, London, 1831).

2. T. B. Curling, "Hydrocele," in Practical Treatise on the Diseases of the Testis and of the Spermatic Cord and Scrotum," (Longmans, Green, London, 1843), pp. 125-181.

3. M. Cutler, "Transillumination as an aid in the diagnosis of breast lesions," Surg. Gynecol. Obstet. 48, 721-729 (1929).

4. K. Shimizu, M. Mouri, and K. Yamamoto, "Trans-body imaging of physiological functions with light," in Optical Methods in Biomedical and Environmental Sciences, H. Ohzu and S. Komatsu, ed. (Elsevier Science, New York, 1994), pp. 63-66.

5. R. S. Balaban and V. A. Hampshire, "Challenges in small animal noninvasive imaging," Inst. Lab. Anim. Res. 42, 248262 (2001).

6. C. Lok, "Picture perfect," Nature 412, 372-374 (2001).

7. D. A. Benaron, "The future of cancer imaging," Cancer Metastasis Rev. 21, 45-78 (2002).

8. T. F. Massoud and S. S. Gambhir, "Molecular imaging in living subjects: seeing fundamental biological processes in a new light," Genes Dev. 17, 545-580 (2003).

9. K. Shimizu and K. Yamamoto, "Imaging of physiological functions by laser transillumination," in Advances in Optical Imaging and Photon Migration, R. R. Alfano and J. G. Fujimoto, eds., Vol. 2 of OSA Trends in Optics and Photonics Series (Optical Society of America, Washington, D.C., 1996), pp. 348352.

10. Y. Taka, K. Sakatani, Y. Kato, and K. Shimizu, "Non-invasive imaging of absorption changes in rat brain by NIR transillumination," Med. Imaging Technol. 17, 545-555 (1999).

11. K. Shimizu and M. Kitama, "Fundamental study on near-axis scattered light and its application to optical computed tomography," Opt. Rev. 7, 383-388 (2000).

12. K. Sakatani, M. Kashiwasake-Jibu, Y. Taka, S. Wang, H. Zuo, K. Yamamoto, and K. Shimizu, "Noninvasive optical imaging of the subarachnoid space and cerebrospinal fluid pathways based on near-infrared fluorescence," J. Neurosurg. 87, 738745 (1997).

13. J. C. Hebden, S. R. Arridge, and D. T. Delpy, "Optical imaging 
in medicine: I. Experimental techniques,” Phys. Med. Biol. 42, 825-840 (1997).

14. S. R. Arridge and J. C. Hebden, "Optical imaging in medicine: II. Modeling and reconstruction,” Phys. Med. Biol. 42, 841-853 (1997).

15. D. J. Hall, J. C. Hebden, and D. T. Delpy, "Evaluation of spatial resolution as a function of thickness for time-resolved optical imaging of highly scattering media," Med. Phys. 24, 361-368 (1997).

16. A. H. Gandjbakhche, R. Nossal, and R. F. Bonner, "Resolution limits for optical transillumination of abnormalities deeply embedded in tissues," Med. Phys. 21, 185-191 (1994).

17. V. Chernomordik, R. Nossal, and A. H. Gandjbakhche, "Point spread functions of photons in time-resolved transillumination experiments using simple scaling arguments," Med. Phys. 23, 1857-1861 (1996)

18. V. Chernomordik, A. H. Gandjbakhche, M. Lepore, R. Esposito, and I. Delfino, "Depth dependence of the analytical expression for the width of the point spread function (spatial resolution) in time-resolved transillumination," J. Biomed. Opt. 6, 441-445 (2001).

19. K. Shimizu, Y. Kato, and K. Tochio, "Improvement of transcutaneous fluorescent image by depth-dependent PSF," in Technical Digest of OSA Biomedical Optics Topical Meetings (CDROM), paper ThF22 (2004).

20. V. Tuchin, Tissue Optics (SPIE, Bellingham, Wash., 2000).

21. A. Ishimaru, Wave Propagation and Scattering in Random Media (Institute of Electrical and Electronics Engineers, New York, 1997).

22. R. C. Benson and H. A. Kues, "Fluorescence properties of Indocyanine Green as related to angiography," Phys. Med. Biol. 23, 159-163 (1978).

23. Institute of Laboratory Animal Resources, Commission on Life Sciences, National Research Council, Guideline for the Care and Use of Laboratory Animals, (National Academy of SciencesNational Research Council, Washington, D.C., 1996). 